\title{
Conexões
}

\section{Práticas corporais como tecnologia social na estratégia saúde da família}

\author{
Sabrina Raquel de Lima de Lima Andrade ${ }^{1}$ \\ Aline Albuquerque Marques ${ }^{2}$ \\ Ana Clécia Silva Ferreira Rodrigues ${ }^{2}$ \\ Neires Alves de Freitas ${ }^{2}$ \\ André Luis Façanha da Silva ${ }^{3}$
}

\section{RESUMO}

O presente relato de experiência tem como objetivo descrever o processo de implantação e a trajetória das Práticas Corporais Comunitárias (PCC) como tecnologia social da Estratégia Saúde da Família (ESF) no município de Sobral/CE. Utilizamos o diário de campo, observação direta nos grupos de práticas corporais em 13 bairros facilitados por profissionais de Educação Física. Nos resultados destacaram-se a predominância de participação do público feminino intergeracional; variação na utilização dos conteúdos da cultura corporal do movimento; espaço de construção de vínculos, autonomia, socialização, criticidade e conhecimentos dos microprocessos de trabalhos para efetivação do apoio matricial nas PCC. A experiência contribuiu para enriquecer os conhecimentos e as necessidades das PCC na ESF no município de Sobral/CE.

Palavras-Chave: Prática corporal. Tecnologia social. Estratégia saúde da família. Educação física.

\footnotetext{
${ }^{1}$ Universidade Estadual do Ceará

${ }^{1}$ Universidade Estadual Vale do Acaraú

${ }^{1}$ Universidade Federal do Ceará

Submetido em: 31 out. 2016

Aceito em: 24 abr. 2017

Contato: sabrina_rla@yahoo.com.br
} 


\section{Body practices as social technology in the strategy family health}

\section{A BStraCT}

This experience report aims to describe the implementation process and the trajectory of the Community Body Practices (PCC) as social technology of the Family Health Strategy (ESF) at Sobral/CE. We used the diary of field, direct observation in the corporal practices groups in 13 neighborhoods facilitated by professionals of Physical Education. The results highlighted the predominance of participation of the intergenerational female audience; Variation in the use of the contents of the movement's body culture; Space for building links, autonomy, socialization, criticality and knowledge of the microprocesses of works to effect the matrix support in the PCCs. The experience contributed to enriching the knowledge and needs of the PCCs in the ESF in the city of Sobral/CE.

Keywords: Body practice. Social technology. Family health strategy. Physical Education.

\section{Prácticas corporales como tecnología social en la estrategia salud de la familia}

\section{RESUMEN}

El presente relato de experiencia tiene como objetivo describir el proceso de implantación y la trayectoria de las Prácticas Corporales Comunitarias (PCC) como tecnología social de la Estrategia Salud de la Familia (ESF) en el municipio de Sobral/CE. Utilizamos el diario de campo, observación directa en los grupos de prácticas corporales en 13 barrios facilitados por profesionales de Educación Física. En los resultados se destacaron la predominancia de participación del público femenino entre generación; variación en la utilización de los contenidos de la cultura corporal del movimiento; espacio de construcción de vínculos, autonomía, socialización, criticidad y conocimientos de los reducidos procesos de trabajos para realización del apoyo matricial en las PCC. La experiencia contribuyó para enriquecer los conocimientos y las necesidades de las PCC en la ESF en el municipio de Sobral/CE.

Palabras Clave: Práctica corporal. Tecnología social. Estrategia salud de la familia. Educación Física. 


\section{INTRODUÇÃO}

As práticas corporais/atividades físicas é um dos temas prioritário da Política Nacional de Promoção da Saúde (PNPS), adjunta ao contexto da ESF, tem objetivo de

[...] promover ações, aconselhamento e divulgação de práticas corporais e atividades físicas, incentivando a melhoria das condições dos espaços públicos, considerando a cultura local e incorporando brincadeiras, jogos, danças populares, entre outras práticas" (BRASIL, 2014a, p. 31-32).

Assim as PCC aproximam-se a configuração de tecnologia social (TS) por potencializar a dimensão subjetiva do participante e as singularidades do grupo, bem como as questões sociais locais.

O Instituto de Tecnologia Social (ITS, 2004) conceitua TS como um conjunto de técnicas, metodologias transformadoras, desenvolvidas e/ou aplicadas na interação com a população e apropriadas por ela, que representam soluções para a inclusão social e melhoria das condições de vida. Levando em consideração o poder das PCC como ferramenta para o desenvolvimento de soluções coletivas, autonomia, criticidade, empoderamento, poder de transformação local na perspectiva de promoção da saúde por meio de metodologias participativas, dialógicas e reflexivas nos espaços coletivos, convergem as PCC a uma configuração de tecnologia social.

Como alternativa para alcançar esse nível, abordagens educativas em meio ao grupo, como a metodologia do círculo de cultura e as rodas de conversas propostos pelo referencial da educação popular, subsidiam a efetivação das PCC no contexto da ESF, levando em consideração o contexto sociocultural dos participantes e envolvendo-os de maneira participativa A educação popular

[...] busca promover a participação dos sujeitos sociais, incentivando a reflexão, o diálogo e a expressão da afetividade, potencializando sua criatividade e sua autonomia. Incorpora a perspectiva do protagonismo dos diversos sujeitos, a valorização das culturas locais nas suas organizações, suas expressões artísticas e as possibilidades de envolvimento de outros setores para o enfrentamento dos problemas cotidianos (DARON, 2014).

Assim, o uso de metodologias ativas permitem mudanças e transformações no modo de promover e diversificar as $\mathrm{PCC}$, e não restringir apenas à ginástica de condicionamento e caminhada orientada. Elas são empregadas para facilitar a interação de pessoas, grupos e organização social focada, segundo (PERERA; GOMES, 2009), no exercício da problematização, poder e de forma democrática. 
As PCC na Atenção Básica devem incluir diversas tecnologias de maneira adequada, conforme as necessidades em saúde. Detemo-nos especialmente as tecnologias leves que caracterizam as relações de produção do acolhimento e vínculo, comunicação, reciprocidade e interação indispensáveis à efetivação do cuidado, tornando-as notórias no território de atuação, sendo essa a essência dos grupos de PCC (MERHY; ONOCKO, 2006).

Cada encontro é mediado por três momentos específicos: acolhimento e aquecimento; desenvolvimento da aula ou produção grupal, baseado nos conteúdos da cultura corporal do movimento; relaxamento e avaliação. Esse é mediado pelo referencial do exercício físico, ludicidade e das técnicas de abordagem grupais (MUNARI; LUCCHESE; MEDEIROS, 2009; DALL'AGNOL et al., 2007).

A educação em saúde dá-se pela aquisição de conhecimento, partilha de informações e apropriação de mundo, o que possibilita os indivíduos de fazerem suas escolhas e firmarem condutas de autocuidado. As PCC, por constituírem uma estratégia de encontro com o outro e com a pluralidade de fatores, favorecem a coprodução de saúde através de elementos da cultura corporal do movimento, considerando as dimensões subjetiva e social do participante, além da sua autonomia (BRASIL, 2010).

A implantação das PCC surge como condicionante para melhorar a qualidade dos serviços de saúde, ocupar os espaços de lazer nos territórios, principalmente os mais vulneráveis e distantes do centro da cidade, promover espaços de reflexão, cuidado, socialização e afetos, visto a insuficiência de políticas públicas de lazer, e o aumento substancial de situações de extrema pobreza, que provavelmente colabore para o aumento da violência e desenvolvimento de agravos. O propósito não é restringir as atividades a um número limitado e um único perfil de sujeitos, mas oferecer as práticas corporais a todos públicos etário, como está posto na PNPS (BRASIL, 2014a).

Debruçar-se sobre esse estudo é de grande relevância por ser considerada uma metodologia inovadora para melhoria das condições de vida da população adscrita nos territórios que dispõem de determinada atividade. Referenciais da educação popular se estabelecem por meio do ato comunicativo, se apresentam nas mais distintas representações da linguagem onde há o desprendimento da conversa formal e técnica para expansão da expressão corporal, da arte, do compartilhamento de vivência e consegue-se, por meio da variedade comunicativa, garantir o desígnio da participação popular no campo da saúde (SOUZA, 2004).

O estudo aludido tem como objetivo descrever o processo de implantação e a trajetória das práticas corporais comunitárias como tecnologia social para a ESF de Sobral/CE. 


\section{Metodologia}

Trata-se de um relato de experiência, de abordagem qualitativa, ilustrando como se deu o processo de implantação de uma tecnologia social no município de Sobral/CE, denominada de PCC nas ESF.

O relato de experiência, características de observação da realidade, não possui a necessidade de testas hipóteses, mas estabelece relações entre os achados da realidade e bases teóricas pertinentes (MACÊDO; MONTEIRO, 2006). Segundo Minayo (2013), a abordagem qualitativa é marcada por significados, valores, crenças e atitudes correspondentes aos participantes, o espaço em que vivem suas condições sociais e dos processos que não se reduzem à operacionalização de variáreis.

A técnica de coleta das informações aconteceu por meio da observação direta, referenciada por Gil (2008) como uma forma onde o pesquisador observa os fatos de maneira espontânea e atua como um expectador para captar as explicações e interpretações do que discorre na realidade. Conjugado a técnica de coleta descrita, o diário de campo, as rodas de conversas e os registros fotográficos enriqueceram as informações investigadas.

Para o diário de campo, Minayo (2013) relata que se compõe de um instrumento para adoção de informações relevantes observadas, como conversas informais, gestos, comportamentos, expressões, hábitos e costumes; conta com informações além das entrevistas formais. Sobre as rodas de conversas, elas possibilitam encontros dialógicos, criando possibilidades de produção e ressignificação de sentido/saberes, ampliando a capacidade do pensar, num movimento contínuo de perceber-refletir-agir-modificar (FREIRE, 2003).

Sobral, cidade onde foi implantada as PCC, está situada na porção noroeste do estado do Ceará, a $232 \mathrm{~km}$ da capital, Fortaleza. É uma cidade considerada de médio porte pelo Instituto Brasileiro de Geografia e Estatística (IBGE, 2015) por possuir uma população estimada de 201.756 de habitantes para o ano de 2015, Possui uma área de unidade territorial de $2.122,897 \mathrm{~km}^{2}$ com densidade demográfica de $88,67 \mathrm{hab} / \mathrm{km}^{2}$ e é uma das cidades com crescente desenvolvimento nos últimos dez anos no Estado.

Em 2007, o projeto de ginástica comunitária foi implementado nos Centros de Saúde da Família (CSF) por profissionais de Educação Física da Residência Multiprofissional em Saúde da Família (RMSF) e/ou do Núcleo de Apoio à Saúde da Família (NASF) e passou a contar com 13 grupos em vários bairros, dentre eles: Alto da Brasília, CAIC, Coelce, COHAB 2, Dom Expedito, Estação, Novo Recanto, Padre Palhano, Pedrinhas, Sinhá Sabóia, Sumaré, Terrenos Novos, Vila União. 
As informações acerca das PCC insurgiram nas rodas da categoria de Educação Física $^{1}$, quando problematizávamos sobre as ações, abordagens e atividades vivenciadas nos grupos. Procede, então, a disposição dos profissionais de Educação Física em averiguar o percurso do surgimento das PCC no município de Sobral/CE, qual parcela da população mais usufrui do serviço, as especificidades da prática e a repercussão dessas na vida dos participantes.

\section{O PERCURSO DA IMPLANTAÇÃO}

As PCC na ESF tiveram sua origem no ano de 2000, com implantação de grupos de caminhada orientada para idosos, na perspectiva da atividade física. Em 2005, iniciase a elaboração da ginástica comunitária, na perspectiva da cultura corporal como uma nova estratégia de ação ampliando à oferta de serviços nos CSF. Surgiu, então, uma iniciativa inovadora de operacionalizar as PCC no cotidiano dos serviços através dos profissionais de Educação Física da $5^{\text {a }}$ turma de RMSF em Sobral. Mas o projeto só foi implantado no ano de 2008, após ser apresentado a sua proposta e os seus objetivos aos gestores e comunidade, embora essa tecnologia tenha angariado recursos do Ministério de Saúde através do edital de incentivo as práticas corporais com concessão do resultado em dezembro de 2006 (SILVA; OLIVEIRA, 2013).

As PCC destinam-se a todas as pessoas pertencentes às comunidades de abrangência dos CSF onde estão inseridos os grupos. Por vezes, os participantes surgem de demanda espontânea e, outras, são encaminhados por profissionais da ESF, visto a inexistência de critérios de exclusão.

Para controle das mesmas, a utilização de mapas de frequência com informações sobre idade, sexo, Agente Comunitário de Saúde (ACS), perfil de saúde são realizados para o controle da participação e busca ativa de participantes faltosos, além de estimular, junto aos ACS, a adesão de mais pessoas à prática. A evolução dos prontuários individuais é reconhecida como uma forma de registro, também, para o conhecimento de outros profissionais da ESF.

$\mathrm{Na}$ atualidade, a TS incorpora elementos da cultura corporal como: dança, esporte, lazer, expressão corporal, reabilitação; e desenvolvem ações com foco na promoção e proteção da saúde, melhoria e qualidade de vida do sujeito, amparado pela evolução histórica-conceitual e social sistematizada, decodificada e reinventada pelo homem.

\footnotetext{
${ }^{1} \mathrm{O}$ espaço da roda é garantido para o desenvolvimento do processo educativo dos profissionais com proposição de fortalecimento da autonomia do sujeito e do coletivo através da práxis. Para Campos (2000), o método da roda é uma tecnologia para o desenvolvimento da co-gestão do coletivo com características administrativa, terapêutica, política e pedagógica. Essa última vem para instigar o estudo e aprendizagem significativa dos profissionais no espaço assegurado para determinada finalidade. Silva; Sousa (2010) consideram a roda de categoria, composta pelos profissionais de Educação Física e preceptoria, no caso, um momento de orientação ao cuidado dos processos pertinentes ao núcleo para construção das práticas e saberes advindos dos territórios por intermédio de alinhamento teórico-conceitual e desenvolvimento de visão perante especificidades territoriais.

Conexões: Educ. Fís., Esporte e Saúde, Campinas: SP, v. 15, n. 3, p. 380-394, jul./set. 2017. ISSN: 1980-9030
} 
O público de presença marcante encontra-se caracterizado, em sua maioria, por mulheres adultas, de jovens à terceira idade, mesmo não havendo critérios de inclusão e/ou exclusão (FIGURA 1). Conforme os dados do Sistema de Informação da Residência Multiprofissional (SIREMU) (SILVA et al., 2015), os grupos de PCC nos territórios existem uma predominância do público já destacado, com participação discreta de homens, perceptível na Figura 2.

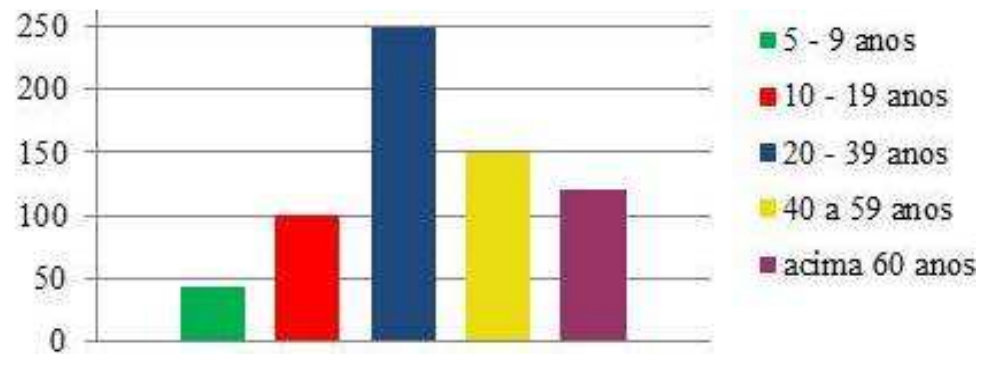

Figura 1 - Distribuição por faixa etária dos participantes dos grupos de práticas corporais.

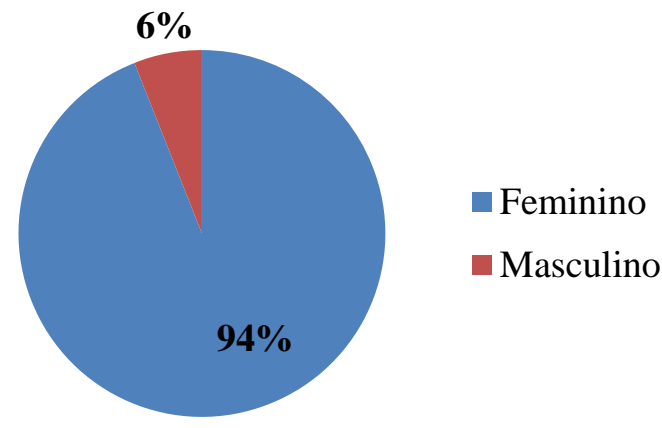

Figura 2 - Caracterização dos participantes por gênero

A Tabela 1 demonstra o quantitativo de participantes a partir dos dados do SIREMU (SILVA et al., 2015), no qual observou um aumento dos participantes de 2009 até o ano de 2014 nas práticas de grupos de caminhada e ginástica comunitária.

Tabela 1- Distribuição cronológica da quantidade de usuários participantes dos grupos de práticas corporais, correspondente ao período de 2009 a 2014, Sobral-Ce

\begin{tabular}{l|c|c|c|}
\hline & $2009 / 2010$ & $2011 / 2012$ & $2013 / 2014$ \\
\hline Grupo de Caminhada & 149 & 155 & 160 \\
\hline $\begin{array}{l}\text { Grupo de Ginástica } \\
\text { Comunitária }\end{array}$ & 201 & 240 & 260 \\
\hline
\end{tabular}

Fonte: SILVA et al., 2015. 
Cada grupo de PCC é acompanhado por um profissional de referência, com formação em Educação Física e/ou vinculado a Equipe de Saúde da Família (EqSF) do CSF. Os momentos de prática acontecem nos equipamentos sociais do bairro como: salão paroquial, praças, igrejas, unidades de saúde, quadras. Além desses espaços há atividades que acontecem na residência de integrantes do grupo, a depender da disponibilidade de equipamentos sociais no território.

O grupo segue um fluxo contínuo para ingresso de novos participantes, a considerar o espaço aberto e sem restrição de pessoas à prática, ainda que leve em consideração os determinantes sociais, como condições socioeconômica, biológica, culturais, religiosos dos participantes. A periodicidade da PCC ocorre entre duas a três vezes por semana, com uma hora de duração cada encontro.

Para condução dos momentos utiliza-se de materiais, como halteres, colchonete, caneleira, bola, faixas elásticas, corda, rede de vôlei e outros materiais alternativos existentes na própria comunidade, como garrafa pet, elástico, canos, saquinho de areia, ligas, cabos de vassoura, caneleiras artesanais e equipamentos para realizar alguns exercícios; todos são adaptados à realidade do território locado.

Como metodologia das aulas, foi verificada a presença de três momentos. No primeiro momento é realizada dinâmica de acolhimento para recepcionar os participantes. Ela serve para se aproximar dos indivíduos, perceber como eles estão chegando naquele dia, seus sentimentos, humores, disposição, inquietações e queixas. Segue-se com uma atividade de aquecimento para adentrar a atividade específica, que pode ser uma brincadeira de energização, como pular corda, uma dança, um alongamento, circuito, jogo ou corridas curtas. A mesma prepara o corpo que estava desacelerado para entrar no ritmo da aula, iniciando um preparo global do corpo.

A segunda parte funciona para desenvolver a atividade, ou seja, aplicam-se as de acordo com o objetivo pré-estabelecido do grupo. No terceiro momento é realizado um relaxamento, seguido de uma avaliação como devolutiva sobre o encontro, havendo relatos de sensações sentidas no corpo e solicitação de sugestões.

Os participantes dessas práticas são encaminhados pela EqSF ou convidados por outros participantes, assim envolvem crianças, adolescentes, jovens, adultos e idosos, uns em minoria comparado à outros. Para complementar as atividades oferecidas nas PCC, uma Avaliação Corporal Integrada (ACI) é realizada logo em seguida que os indivíduos entram no grupo, e possui frequência entre seis a oito meses para reavaliação.

A ACI é realizada para colher dados e informações sobre a história de vida, condicionantes e determinantes da saúde. O monitoramento de aspectos epidemiológicos, contemplando características socioeconômicas e culturais dos 
participantes das PCC é primordial para subsidiar o planejamento das atividades a serem realizadas no grupo de acordo com as características colhidas. Em seguida, essa avaliação é encaminhada para o setor de vigilância epidemiológica onde tem o Sistema de Informação e Monitoramento da Avaliação Corporal Integrada (SIMACI). O sistema, além de hospedar dados, é muito útil para conhecer o perfil dos participantes e do grupo, pois contribui no planejamento das atividades, considerando todos os aspectos individuais e coletivos (SILVA; DAMIANI, 2005).

Esse encontro entre o profissional de Educação Física e o participante possibilita ir além do momento avaliativo, pois podemos perceber outras necessidades de saúde e como viabilizar soluções conjuntas e respostas para tal problema. Ainda colaboram para identificar os avanços, impactos no participante e nos grupos, percebendo os olhares implicados com a ação e remodelar conforme as proposições.

Para complementar a oferta assistencial, dispomos da preceptoria, caracterizada por um profissional com expertise e formação para lidar com os processos das PCC na ESF e pertencente ao corpo docente do Sistema Municipal Saúde-Escola. Ele faz-se presente como uma estratégia de apoio pedagógico no processo de ensino-aprendizagem aos profissionais de Educação Física presidindo orientações do trabalho da equipe, se efetivando na perspectiva do diálogo e na busca de juntos formularem alternativas de encaminhamentos que possam ajudar na solução adequada dos problemas (SOUZA, 2004).

\section{Discussão}

As PCC na ESF estão disponíveis para os diferentes ciclos de vida com o intuito de promover espaços de cuidado, através de ações de prevenção de doenças e promoção da saúde de forma intergeracional, atentos às necessidades individuais e coletivas de forma integral, abrangendo a população com os princípios da organização e desenvolvimento das práticas corporais/atividades físicas, preconizadas nas Diretrizes do NASF (BRASIL, 2010).

Quando observamos as atividades mais praticadas conforme o sexo constata-se que no estudo de Linhares; Silva (2014) os homens têm predominância pela musculação, futebol, lutas, caminhada e corrida; enquanto as mulheres aderem mais a ginástica, a caminhada, a dança e a hidroginástica, corroborando com os dados da pesquisa em questão. Todavia, as PCC no município ainda não oferecem a maioria das atividades de interesse ao público masculino, refletindo na pouca adesão desse gênero e evidenciando a necessidade da inserção de determinadas práticas nos grupo, com a finalidade de incentivar o usufruto de toda a comunidade. Do outro lado, é perceptível a satisfação das mulheres em poderio nas PCC, como bem demostrado na Figura 1. 
Destacamos que, apesar da baixa oferta de atividades preferenciais do público masculino, há variação com os elementos da cultura corporal do movimento possibilitando aos participantes o despertar para sensações, percepções, potencialidades e limites com o corpo, a partir das vivências facilitadas em grupo. Dessa maneira, a visão de corpo, como uma condição instrumentalista, pode ser transformada no intuito de ampliar o olhar sobre as práticas, considerar a dimensão subjetiva no ato de cuidar e perceber que este corpo é carregado de afetos, experiências de vida e sensações (BILIBIO; CECCIM, 2007).

Para Furtado et al. (2016), as PCC inseridas na ESF como um dos serviços ofertados se caracterizam como um espaço de cuidado, promoção de saúde e de transformação social, pois para além do cuidado com o corpo é também um espaço para reflexões, socialização, promoção de vínculos, afetos e inserção social. Busca privilegiar o sujeito ao invés da doença, e, é por meio das práticas que os profissionais buscam dar outro significado a visão de corpo, no intuito de tencionar os sujeitos a compreender a sua existência para além das questões orgânicas.

Logo, as PCC, na perspectiva da promoção da saúde, representa uma estratégia que possibilita situações favoráveis para o desenvolvimento das condições de saúde, contribuindo assim para o crescimento pessoal dos indivíduos; para além dos elementos referidos, as práticas tornam-se coadjuvantes no processo de cuidado do corpo, pois constroem relações de vínculos e de corresponsabilidade (CARVALHO, 2006).

As PCC sistemáticas fixam-se na ESF na forma de programas de práticas corporais/atividades físicas, a fim de dissociar o binômio sedentarismo/doença e associar atividade física/saúde, mesmo sabendo que o público demanda de necessidades individuais e não é imposta restrição para quem tem o interesse de participar (PONTES; CHAGAS, 2010). No alcance às necessidades dos participantes, as categorias inerentes à EqSF e a equipe multiprofissional adentram as PCC com o desejo de contribuir com seu núcleo dentro dos processos de trabalho.

Na lógica de refletir repetidamente o processo de trabalho nos CSF, mudanças nos microprocessos de trabalho, (re) pensando as práticas em saúde a partir da leitura ampliada da realidade de vida dos indivíduos, garantem que as intervenções se deem em consonância com as singularidades de cada um deles, se consolide na integralidade da assistência, na reorganização tecnológica a partir das tecnologias leves e na clínica ampliada com destaque ao espaço grupal das práticas corporais (BONFADA et al., 2012).

Percebemos através das observações realizadas no âmbito das PCC, que a abordagem grupal precisa de uma maior organicidade para seu desenvolvimento e que contemple a participação, o diálogo, o planejamento, a criatividade e a experiência prévia dos participantes. 
A presença de equipes multiprofissionais do NASF e RMSF, como já citado, onde se encontram os profissionais de Educação Física, se configura como uma política nacional instituída de categorias diversas, sendo eles Assistentes Sociais, Profissional de Educação Física, Farmacêutico, Fisioterapeuta, Fonoaudiólogo, Nutricionista, Psicólogo, Terapeuta Ocupacional em trabalho agrupado à EqSF para oferecer apoio de núcleo e campo nas PCC.

No CSF, contamos com a colaboração dos profissionais que compõem as EqSF, como médico, enfermeiro(a), ACS, odontólogos orientando os usuários para adesão e/ou permanência nas PCC, por meio de contribuições pedagógicas sobre o processo saúde-doença e cuidados ininterruptos a serem tomados.

Peduzzi (2001) considera o trabalho coletivo, exemplificando o trabalho em equipe multiprofissional, a ser construído por meio de relação recíproca, de dupla mão, entre as múltiplas intervenções técnicas e a interação dos profissionais de diferentes áreas, configurando, através da comunicação, a articulação das ações e a cooperação como uma forma de assistir melhor os indivíduos.

Essa interação de EqSF com equipes ou profissionais com outros núcleos de conhecimentos diferentes dos profissionais da ESF se configura como um apoio matricial. A interação deve dar-se a partir das necessidades, dificuldades, ou limites da EqSF diante demandas e necessidades de saúde, ao mesmo tempo contribuindo para ampliar a capacidade do cuidado e o escopo de oferta de serviço para garantir a continuidade do cuidado ao indivíduo. A partilha de conhecimentos oportuniza uma maior colaboração no núcleo e solidificação de um campo preparado para receber os participantes das PCC (BRASIL, 2014b; FIGUEIREDO; CAMPOS, 2009).

O apoio matricial apresenta dimensões de suporte, sendo elas, assistencial e técnico-pedagógico. A dimensão assistencial é aquela que vai produzir ação clínica direta com os usuários, e a ação técnico-pedagógica vai produzir ação de apoio educativo com e para a equipe. Essas duas dimensões podem e devem fundir-se nas PCC, concedendo à equipe multiprofissional e a EqSF capacidade apoiar os grupos e até chegar a facilitar momentos de PCC (BRASIL, 2014b).

Os profissionais de apoio desempenham atribuições que transcendem a dimensão de assessoria para a ideia de acompanhamento pedagógico colaborando com suas expertises voltadas sobre os referenciais que orientam a práxis e os demais no apoio na realização de planejamentos, execução e avaliação das PCC.

Com a colaboração de profissionais da EqSF e equipe multiprofissional nas PCC, os participantes expressam contentamento, pois um olhar múltiplo aumenta o leque de cuidado e, consequentemente, demandas de núcleo que não chegariam ao profissional específico para resolução do problema passam a ser facilitadas pelos 
mesmos estarem como apoio ao grupo. Ocasiões como essa estão sendo frequentes nas PCC desde sua implantação formalizando o núcleo da Educação Física como um campo de oportunidades para o desabrochar de outras categorias, sem esquecer do fortalecimento que a Educação Física passa a ter quando desfruta do trabalho conjunto nas PCC.

\section{CONSIDERAÇÕES Finais}

Resultante do relato de experiência tornou-se evidente a relevância das PCC, no município do Sobral/CE, para a dimensão orgânica e o destaque do fortalecimento da autonomia, da subjetividade, da criticidade enquanto sujeitos políticos e cidadãos, bem como da comunidade em geral e grupos vulneráveis, como destacado por Brasil (2014b).

Como um dos eixos principais da PNPS, as práticas corporais/atividades físicas alcança seu objetivo de promover ações divulgando as PCC, incentivando a melhoria das condições dos espaços públicos e considerando a cultura local para incorporação de elementos da cultura corporal do movimento. Essa configuração abrange um quantitativo de participantes considerável para caracterizar as práticas como uma TS, pois a população se apropria das PCC como meio de transformação e melhoria das condições de vida. Para conquistar uma maior adesão dos participantes do sexo masculino, ainda em minoria, seria primordial uma busca ativa bem próxima a eles, assim como a inserção de práticas que os pudessem conquistar para torná-los assíduos.

Há outra, é necessário pensar em estratégias para a sustentabilidade das PCC, pois, com a alta rotatividade de profissionais, ter somente um facilitador de referência não prioriza a continuidade do cuidado e o vínculo existente entre profissional/facilitador e indivíduo/participante. Nesse caso, o apoio matricial garante a perpetuidade das ações grupais reduzindo as oscilações possivelmente ocorridas no espaço.

\section{REFERÊNCIAS}

BILIBIO, Luiz Fernando; CECCIM, Ricardo Burg. Singularidades da educação física na saúde: desafios à educação de seus profissionais e ao matriciamento interprofissional. In: FRAGA, Alex Branco; WACHS, Felipe (Org.). Educação física e saúde coletiva: políticas de formação e perspectivas de intervenção. Porto Alegre: Ed. da UFRGS, 2007. p. 47-62. 
BONFADA, Diego et al. A integralidade da atenção à saúde como eixo da organização tecnológica nos serviços. Ciência \& Saúde Coletiva, Rio de Janeiro, v.17, n. 2, p. 555$560,2012$.

BRASIL. Ministério da Saúde. Diretrizes do NASF: Núcleo de Apoio Saúde da Família. Cadernos de Atenção Básica, n. 27. Brasília, 2010.

BRASIL. Ministério da Saúde. Portaria nº 2.446, de 11 de novembro de 2014. Redefine a Política Nacional de Promoção da Saúde. Diário Oficial da União, Brasília, n 220, p. 68, 13 nov. 2014a. Seção 1.

BRASIL. Ministério da Saúde. Núcleo de Apoio à Saúde da Família. Cadernos de Atenção Básica, n. 39. Brasília, 2014b.

INSTITUTO BRASILEIRO DE GEOGRAFIA E ESTATÍSTICA (IBGE). Pesquisa Nacional por Amostra de Domicílios.

<http://www.cidades.ibge.gov.br/xtras/perfil.php?lang=\&codmun=231290\&search=cea ra|sobral.> Acesso em: 20 abr. 2015.

CAMPOS, Sousa Wagner Gastão. Um método para análise e co-gestão de coletivos: a construção do sujeito, a produção de um valor de uso e a democracia em instituições: $\mathrm{O}$ método da roda. São Paulo: Hucitec, 2000.

CARVALHO, Yara Maria. Promoção da saúde, práticas corporais e atenção básica. In: BRASIL. Revista Brasileira Saúde da Família, Brasília, p. 33-45, 2006.

DALL'AGNOL, Clarice Maria et al. O trabalho com grupos como instância de aprendizagem em saúde. Revista Gaúcha Enfermagem, Rio Grande do Sul, v. 28, n. 1, p. 21-26, 2007.

DARON, Vanderléia. A educação popular em saúde como referencial para as nossas práticas na saúde. In: BRASIL. Ministério da Saúde. II Caderno de educação popular em saúde. Brasília, 2014. p. 123-146.

FIGUEIREDO, Mariana Dorsa; CAMPOS, Rosana Onocko. Saúde mental na atenção básica à saúde de Campinas, SP: uma rede ou um emaranhado? Ciências \& Saúde Coletiva, Rio de Janeiro, v.14, n.1, p.129-138, 2009. Disponível em:

<http://dx.doi.org/10.1590/S1413-81232009000100018>. Acesso em: 06 out. 2015.

FREIRE, Paulo. Pedagogia da esperança: um reencontro com a pedagogia do oprimido. Rio de Janeiro: Paz e Terra; 2003. 
FURTADO, Roberto Pereira et al. Educação física e saúde mental: uma análise da rotina de trabalho dos profissionais dos CAPS de Goiânia. Movimento, Porto Alegre, v. 22, n. 4, 1077-1090, out./dez. 2016.

GIL, Antônio Carlos. Como elaborar projetos de pesquisa. São Paulo: Atlas, 2008.

INSTITUTO DE TECNOLOGIA SOCIAL (ITS). Reflexões sobre a construção do conceito de tecnologia social. In: DE PAULO, Antônio et al. Tecnologia social: uma estratégia para o desenvolvimento. Rio de Janeiro: Fundação Banco do Brasil, 2004.

LINHARES, Anagécia Sousa; SILVA, André Luis Façanha. Caracterização do grupo de práticas corporais na Atenção Primária à Saúde em Sobral - Ceará. SANARE, Sobral, v. 13, n.1, p.56-63, jan./jun. 2014.

MACÊDO, Virgílio Cesar Dourado de; MONTEIRO, Ana Ruth Macêdo. Educação e saúde mental na família: experiência com grupos vivenciais. Texto \& Contexto Enfermagem, Florianópolis, v.15, n. 2, p. 222-230, abr./jun. 2006.

MERHY, Emerson Elias; ONOCKO, Rosana. Agir em saúde: um desafio para o público. São Paulo: Hucitec, 2006.

MINAYO, Maria Cecília Souza. O desafio do conhecimento: pesquisa qualitativa em saúde. São Paulo: Hucitec, 2013.

MUNARI, Denize Bouttelet; LUCCHESE, Roselma; MEDEIROS, Marcelo. Reflexões sobre o uso de atividades grupais na atenção a portadores de doenças crônicas. Ciências, Cuidado e Saúde, Maringá, v. 8, supl. 8, p. 148-54, 2009. Disponível em:

<http://www.periodicos.uem.br/ojs/index.php/CiencCuidSaude/article/view/9742>.

PEDUZZI, Marina. Equipe multiprofissional de saúde: conceito e tipologia. Revista de Saúde Pública, São Paulo, v. 35, n. 1, p. 103-109, 2001. Disponível em: <http://www.scielo.br/pdf/rsp/v35n1/4144.pdf>. Acesso em: 3 mar. 2015.

PERERA, Apes Falcão; GOMES, João Carlos Costa. O uso de metodologias participativas na democratização do conhecimento: Avaliação de redes de referências na região Sul do Rio Grande do Sul. Revista Extensão Rural, Rio Grande do Sul, ano 16, n. 18, jul./dez. 2009.

PONTES, Flávia Campos; CHAGAS, Maristela Inês Osawa. Ginástica comunitária na Estratégia Saúde da Família do município de Sobral-CE: avaliação a partir dos discursos dos profissionais e usuários. 2010. Trabalho de Conclusão de Curso (Especialização) - Universidade Estadual Vale do Acaraú, Sobral, 2010. 
SILVA, Ana Márcia; DAMIANI, Iara Regina. As práticas corporais na contemporaneidade: pressupostos de um campo de pesquisa e intervenção social. In: SILVA, Ana Márcia; DAMIANI, Iara Regina (Org.). Práticas corporais: gênese de um movimento investigativo em educação física. Florianópolis: Nauemblu Ciência \& Arte: 2005. v. 1.

SILVA, André Luis Façanha; SOUSA, Roberta Menezes. A roda como espaço de cogestão da residência multiprofissional em saúde da família do município de Sobral CE. SANARE, Sobral, v. 9, n. 2, p. 07-13, jul./dez. 2010.

SILVA, André Luis Façanha; OLIVEIRA, Bráulio Nogueira. A trajetória da educação física no SUS em Sobral-CE: um resgate histórico. Conexões: Revista da Faculdade de Educação Física da UNICAMP, Campinas, v. 11, n. 2, p. 193-207, 2013.

SILVA, André Luis Façanha et al. SIREMU: uma tecnologia de gestão e formação de processos de trabalho na Estratégia Saúde da Família. Prêmio InovaSUS 2012/2013: valorização de boas práticas e inovação na gestão do trabalho na saúde. Brasília: Ministério da Saúde, 2015.

SOUZA, José Nicolau. Prática docente comprometida e o apoio didático pedagógico no ensino superior: a experiência da FARN. Revista da FARN: Revista da Faculdade Natalense, Natal, v. 3, n. 1/2, p. 209-221, jul. 2003/jun. 2004. 